Journal for ImmunoTherapy of Cancer

\section{Phase IA/IB study of single-agent tislelizumab, an investigational anti- PD-1 antibody, in solid tumors}

Jayesh Desai, ${ }^{1}$ Sanjeev Deva, ${ }^{2}$ Jong Seok Lee, ${ }^{3}$ Chia-Chi Lin, ${ }^{4}$ Chia-Jui Yen, ${ }^{5}$ Yee Chao, ${ }^{6}$ Bhumsuk Keam, ${ }^{7}$ Michael Jameson, ${ }^{8}$ Ming-Mo Hou, ${ }^{9}$ Yoon-Koo Kang, ${ }_{7}^{10}$ Ben Markman, ${ }_{11}$ Chang-Hsien Lu, ${ }^{12}$ Kun-Ming Rau, ${ }^{13}$ Kyung-Hun Lee, ${ }^{7}$ Lisa Horvath, ${ }^{14,15}$ Michael Friedlander, ${ }^{16}$ Andrew Hill, ${ }^{17}$ Shahneen Sandhu, ${ }^{1}$ Paula Barlow, ${ }^{2}$ Chi-Yuan Wu, ${ }^{18}$ Yun Zhang, ${ }^{19}$ Liang Liang, ${ }^{19}$ John Wu, ${ }^{18}$ Virginia Paton, ${ }^{18}$ Michael Millward ${ }^{20}$

\section{ABSTRACT}

To cite: Desai J, Deva S, Lee JS, et al. Phase IA/IB study of single-agent tislelizumab, an investigational anti-PD-1 antibody, in solid tumors. Journal for ImmunoTherapy of Cancer 2020;8:e000453. doi:10.1136/jitc-2019-000453

- Additional material is published online only. To view, please visit the journal online (http://dx.doi.org/10.1136/jitc2019-000453).

Accepted 23 April 2020

Check for updates

(C) Author(s) (or their employer(s)) 2020. Re-use permitted under CC BY-NC. No commercial re-use. See rights and permissions. Published by BMJ.

For numbered affiliations see end of article.

Correspondence to

Dr Jayesh Desai;

Jayesh.Desai@petermac.org tumors.
Background The programmed cell death-1/programmed cell death ligand-1 (PD-1/PD-L1) axis plays a central role in suppressing antitumor immunity; axis dysregulation can be used by cancer cells to evade the immune system. Tislelizumab, an investigational monoclonal antibody with high affinity and binding specificity for PD-1, was engineered to minimize binding to FcyR on macrophages to limit antibody-dependent phagocytosis, a potential mechanism of resistance to anti-PD-1 therapy. The aim of this phase IA/IB study was to investigate the safety/ tolerability, antitumor effects and optimal dose and schedule of tislelizumab in patients with advanced solid

Methods Patients (aged $\geq 18$ years) enrolled in phase IA received intravenous tislelizumab $0.5,2,5$ or $10 \mathrm{mg} /$ kg every 2 weeks; 2 or $5 \mathrm{mg} / \mathrm{kg}$ administered every 2 weeks or every 3 weeks; or $200 \mathrm{mg}$ every 3 weeks; patients in phase IB received $5 \mathrm{mg} / \mathrm{kg}$ every 3 weeks. Primary objectives were to assess tislelizumab's safety/ tolerability profile by adverse event (AE) monitoring and antitumor activity using RECIST V.1.1. PD-L1 expression was assessed retrospectively with the VENTANA PD-L1 (SP263) Assay.

Results Between May 2015 and October 2017, 451 patients $(n=116, I A ; n=335$, IB) were enrolled. Fatigue $(28 \%)$, nausea $(25 \%)$ and decreased appetite $(20 \%)$ were the most commonly reported AEs. Most AEs were grade 1-2 severity; anemia (4.9\%) was the most common grade 3-4 AE. Treatment-related AEs led to discontinuation in $5.3 \%$ of patients. Grade 5 AEs were reported in 14 patients; 2 were considered related to tislelizumab. Pneumonitis $(2 \%)$ and colitis $(1 \%)$ were the most common serious tislelizumab-related AEs. As of May 2019, 18\% of patients achieved a confirmed objective response in phase IA and $12 \%$ in phase IB; median follow-up duration was 13.6 and 7.6 months, respectively. Pharmacokinetics, safety and antitumor activity obtained from both phase IA and IB determined the tislelizumab recommended dose; ultimately, tislelizumab $200 \mathrm{mg}$ intravenous every 3 weeks was the dose and schedule recommended to be taken into subsequent clinical trials.

Conclusions Tislelizumab monotherapy demonstrated an acceptable safety/tolerability profile. Durable responses were observed in heavily pretreated patients with advanced solid tumors, supporting the evaluation of tislelizumab $200 \mathrm{mg}$ every 3 weeks, as monotherapy and in combination therapy, for the treatment of solid tumors and hematological malignancies.

Trial registration number NCT02407990.

\section{INTRODUCTION}

The programmed cell death-1/programmed cell death ligand-1 (PD-1/PD-L1) axis plays a central role in suppressing antitumor immunity; dysregulation of the PD-1/PD-L1 axis can be used by cancer cells to evade the immune system. ${ }^{12}$ PD-L1 is an immune checkpoint protein that is often overexpressed on the surface of tumor and immune cells in the tumor microenvironment. ${ }^{3}{ }^{4}$ PD-1, the cell receptor for PD-L1, is mainly expressed in activated $\mathrm{T}$ cells. ${ }^{5}$ An increase in PD-1 expression in the tumor microenvironment has been reported in many cancer types. ${ }^{6-8}$ Increased expression of PD-1 and PD-L1 is often associated with poor survival but may be predictive of anti-PD-1/PD-L1 antitumor activity. $^{9-11}$

Tislelizumab is an investigational humanized IgG4 monoclonal antibody with high affinity and binding specificity for PD-1. ${ }^{12}$ Tislelizumab was engineered to minimize binding to $\mathrm{Fc} \gamma \mathrm{R}$ on macrophages in order to limit antibody-dependent phagocytosis, a potential mechanism of resistance to antiPD-1 therapy. ${ }^{1}$ Preclinical data suggest tislelizumab does not bind to FcyRI, whereas other anti-PD-1 antibodies bind to Fc $\gamma \mathrm{RI}$ in a manner consistent with human IgG4 antibody affinity. $^{12}$ Furthermore, in cell-based assays, tislelizumab enhanced the functional activity of human $\mathrm{T}$ cells and pre-activated primary peripheral blood mononuclear cells. ${ }^{12}$ 


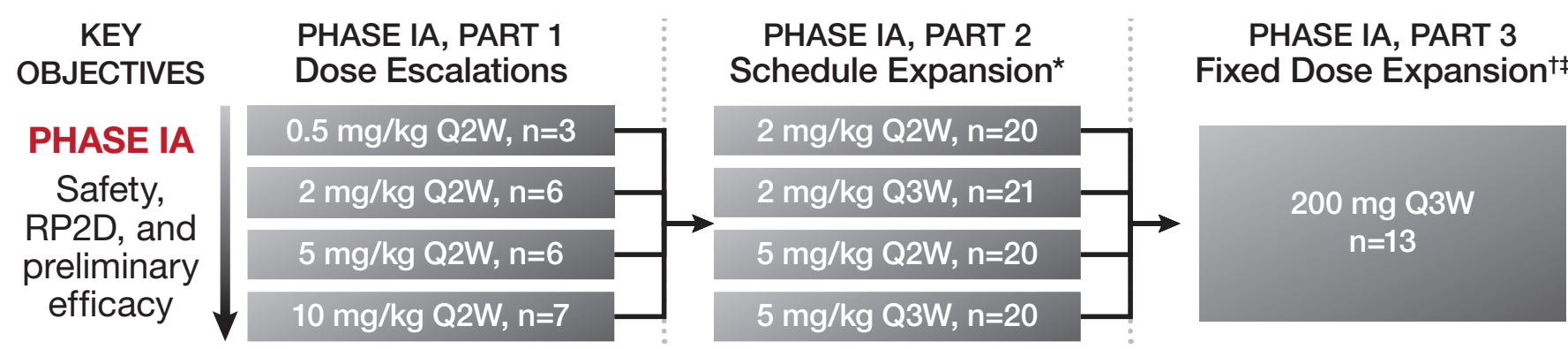

$5 \mathrm{mg} / \mathrm{kg}$ Q3W

PHASE IB: Indication Expansion

\section{Expansion in 330 patients with multiple tumor types}

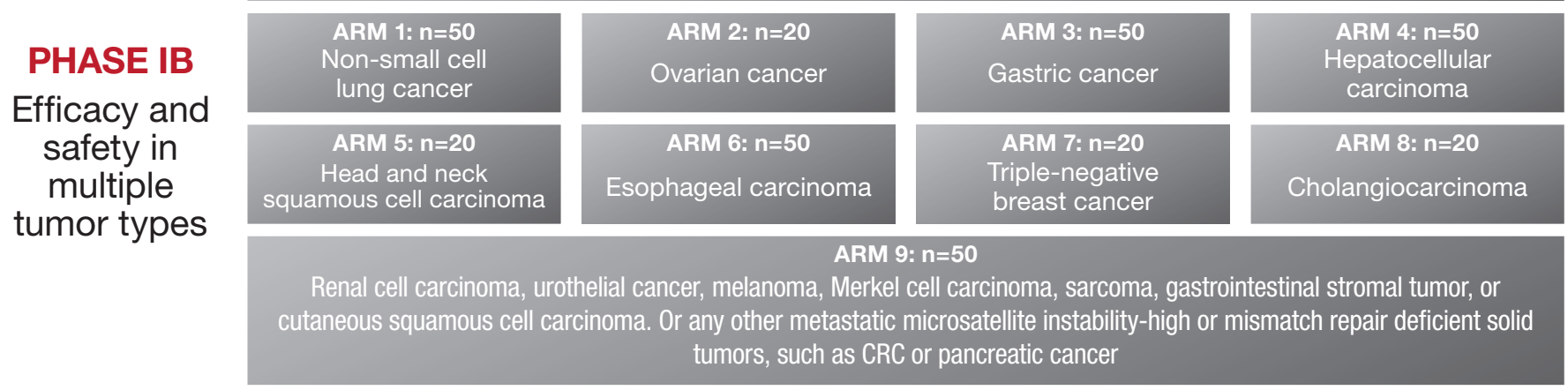

Figure 1 Study design. CRC, colorectal cancer; Q3W, every 3 weeks.

This first-in-human (FIH), dose-escalation/doseexpansion study assessed the safety/tolerability, pharmacology and clinical activity of tislelizumab in patients with advanced solid tumors. The primary objective was to evaluate the safety and tolerability of tislelizumab (phase IA), as well as the antitumor response (phase IB). Secondary end points included determining the maximum tolerated dose (MTD) and the optimal dose and treatment regimen. Confirmed objective response rate (ORR) to tislelizumab by PD-L1 status was an exploratory end point.

\section{METHODS}

Study design and treatment administration

The design of this phase IA/IB study is detailed in figure 1. Phase IA comprised three parts. Part 1 was a dose-escalation/dose-finding phase that followed a modified 3+3 design. Four weight-based dose levels $(0.5,2$, 5 and $10 \mathrm{mg} / \mathrm{kg}$ every 2 weeks) were assessed for doselimiting toxicity (DLT). If a DLT was observed in the first three patients completing one cycle of treatment at a given dose level, three additional patients were enrolled. A DLT was defined as an adverse event (AE) occurring in the first 28-day cycle that met the predefined criteria based on grading per NCI-CTCAE V.4.03. DLT criteria included any grade $\geq 4$ non-hematological toxicity or grade 4 laboratory anomaly irrespective of duration; any grade $\geq 3$ immune-related $\mathrm{AE}$ (irAE), grade 3 tumor flare (ie, local pain, irritation or rash localized at sites of known/suspected tumor) for $>7$ days; grade 3 AEs irrespective of duration, except for laboratory abnormalities, diarrhea, nausea and vomiting; asymptomatic biochemical abnormalities that improve to grade $\leq 2$ within 3 days of institution of supportive care and grade 2 ophthalmological toxicities. Hematological toxicities meeting DLT criteria include febrile neutropenia (defined as absolute neutrophil count $<1000 / \mathrm{mm}^{3}$ with a single temperature of $38.3^{\circ} \mathrm{C}$ or a sustained temperature of $38^{\circ} \mathrm{C}$ for $>1$ hour); grade 4 thrombocytopenia, grade 4 anemia or grade 4 neutropenia lasting $>7$ days; grade 3 thrombocytopenia with bleeding and grade 3 neutropenic infection. No additional patients were required if a DLT was observed in one of six patients. Dose escalation continued until two out of six patients in each dose cohort experienced a DLT. If an MTD could not be established after evaluation of all planned doses, then the dose for subsequent studies would be determined based on safety and pharmacokinetic (PK) profile from aggregated data across the study. Part 2 of phase IA was a schedule expansion that evaluated tislelizumab at 2 and $5 \mathrm{mg} / \mathrm{kg}$ every 2 weeks and every 3 weeks. Part 3 of phase IA (fixed-dose expansion) evaluated tislelizumab $200 \mathrm{mg}$ administered every 3 weeks. The phase IB component of this study was an expansion phase of tislelizumab at $5 \mathrm{mg} / \mathrm{kg}$ every 3 weeks in nine diseasespecific cohorts. The initial administration of tislelizumab was administered via a 60 min infusion, if tolerated, the infusion was shortened to $30 \mathrm{~min}$ for all subsequent administrations. Dose modifications/delays were allowed; criteria for dose modifications are presented on page 2 of the online supplementary appendix. 
Table 1 Patient demographics and baseline disease characteristics

\begin{tabular}{|c|c|c|c|}
\hline & $\begin{array}{l}\text { Phase IA } \\
(n=116)\end{array}$ & $\begin{array}{l}\text { Phase IB } \\
(n=335)\end{array}$ & $\begin{array}{l}\text { Overall } \\
(n=451)\end{array}$ \\
\hline Age (median years)* & 60.5 (19.0 to 80.0$)$ & 61.0 (18.0 to 81.0$)$ & 61.0 (18.0 to 81.0$)$ \\
\hline Male & $55(47.4)$ & $191(57.0)$ & $246(54.5)$ \\
\hline Female & $61(52.6)$ & $144(43.0)$ & $205(45.5)$ \\
\hline African-American & $0(0.0)$ & $5(1.5)$ & $5(1.1)$ \\
\hline Caucasian & $101(87.1)$ & $189(56.4)$ & $290(64.3)$ \\
\hline Other & $5(4.3)$ & $18(5.4)$ & $26(5.7)$ \\
\hline \multicolumn{4}{|l|}{ ECOG performance status } \\
\hline $0 \ddagger$ & $18(16.4)$ & $50(15.6)$ & $68(15.8)$ \\
\hline 1 & $33(30.0)$ & $119(37.2)$ & $152(35.3)$ \\
\hline 2 & $22(20.0)$ & $77(24.1)$ & $99(23.0)$ \\
\hline$\geq 3$ & $37(33.6)$ & $74(23.1)$ & $111(25.8)$ \\
\hline Prior radiotherapy & $63(54.3)$ & $178(53.1)$ & $241(53.4)$ \\
\hline Prior anticancer surgery & $100(86.2)$ & $223(66.6)$ & $323(71.6)$ \\
\hline
\end{tabular}

Data presented as $\mathrm{n}(\%)$, except where noted.

*Data presented as median (range).

†Only therapy used for metastatic, locally advanced or palliative is counted as a line of systemic therapy. The ' $n$ ' is number of patients with any line of prior systemic therapies.

$\ddagger$ Patients may have received adjuvant and/or neoadjuvant therapies.

ECOG, Eastern Cooperative Oncology Group.

Approximately 120 patients were planned to be enrolled in phase IA. Twenty-four patients were needed for part 1, and 20 patients were planned for the schedule-expansion and fixed-dose cohorts. Approximately 330 patients were planned to be enrolled in phase IB in the nine diseasespecific cohorts.

\section{Study population}

Patients (aged $\geq 18$ years) with previously treated histologically/cytologically confirmed advanced solid tumors with measurable disease (as defined by Response Evaluation Criteria in Solid Tumors (RECIST) V.1.1 criteria), an Eastern Cooperative Oncology Group performance status $\leq 1$ and adequate organ function, were eligible for enrollment. Patients with previously treated brain metastases that was/were asymptomatic and radiographically stable and not requiring steroid medications for 4 weeks prior to enrollment were also eligible. Patients who received prior anti-PD-1/PD-L1 treatments; had a history of hypersensitivity reactions to monoclonal antibodies; a history of, or active, autoimmune disease; a history of interstitial lung disease or non-infectious pneumonitis, except for radiation-induced cases; a prior malignancy within the previous 2 years except for locally curable cancers that have apparently been cured (eg, basal or squamous cell skin cancer, superficial bladder cancer or carcinoma in situ of the cervix or breast cancer); patients who had prior liver transplant, allogeneic organ transplantation or bone marrow transplant and patients with a requirement for systemic treatment with corticosteroids $(<10 \mathrm{mg}$ daily prednisone equivalents) or other immunosuppressive medications within 2 weeks of study drug administration were excluded. PD-L1 expression was assessed retrospectively.

\section{Study objectives and assessments}

The primary objectives of this study were to assess the safety and tolerability of tislelizumab (phase IA) as well as antitumor response (phase IB). Secondary objectives included determining the MTD and the optimal dose and treatment regimen, characterization of the tislelizumab PK profile and assessment of host immunogenicity to tislelizumab; assessment of confirmed ORR by PD-L1 status was exploratory.

Safety/tolerability was assessed across the entire study and included continuous monitoring of AEs, as well as vital signs, physical examinations, electrocardiograms and laboratory investigations at specific study visits. AEs were 


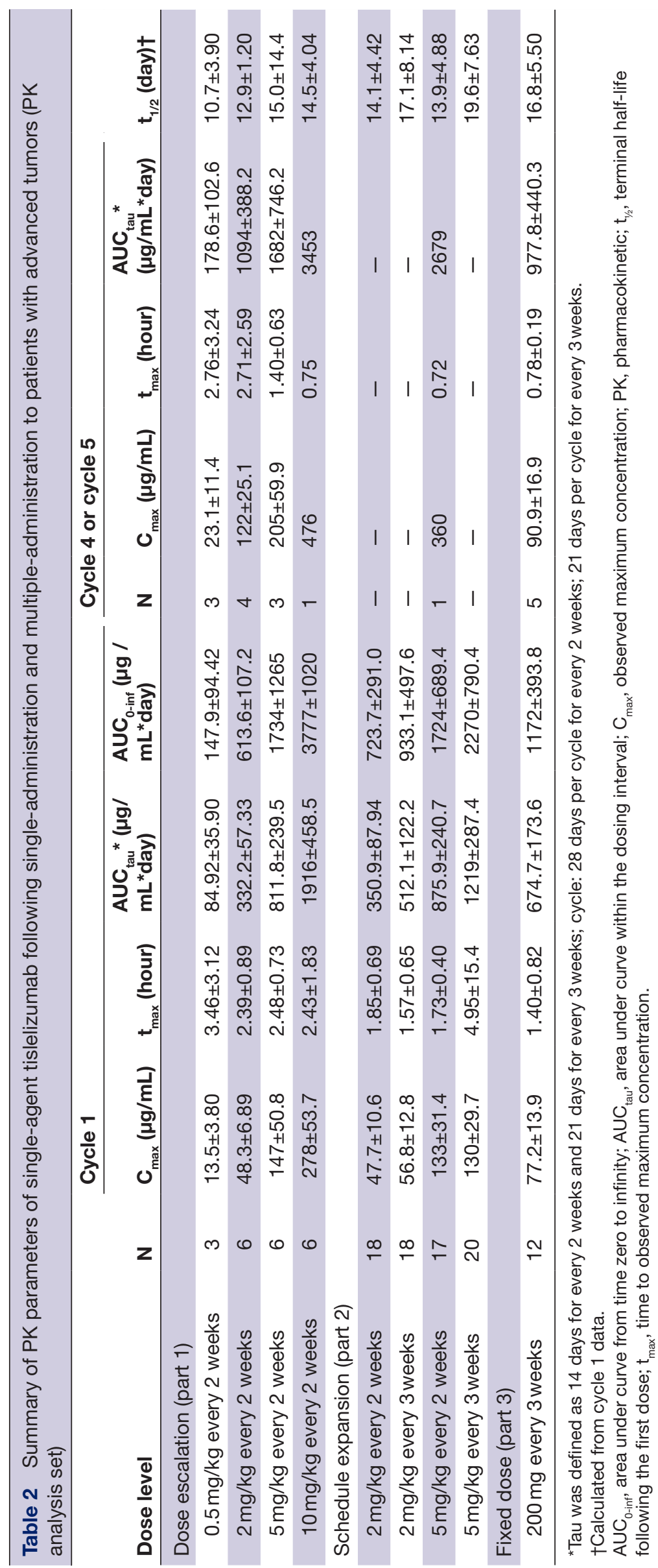




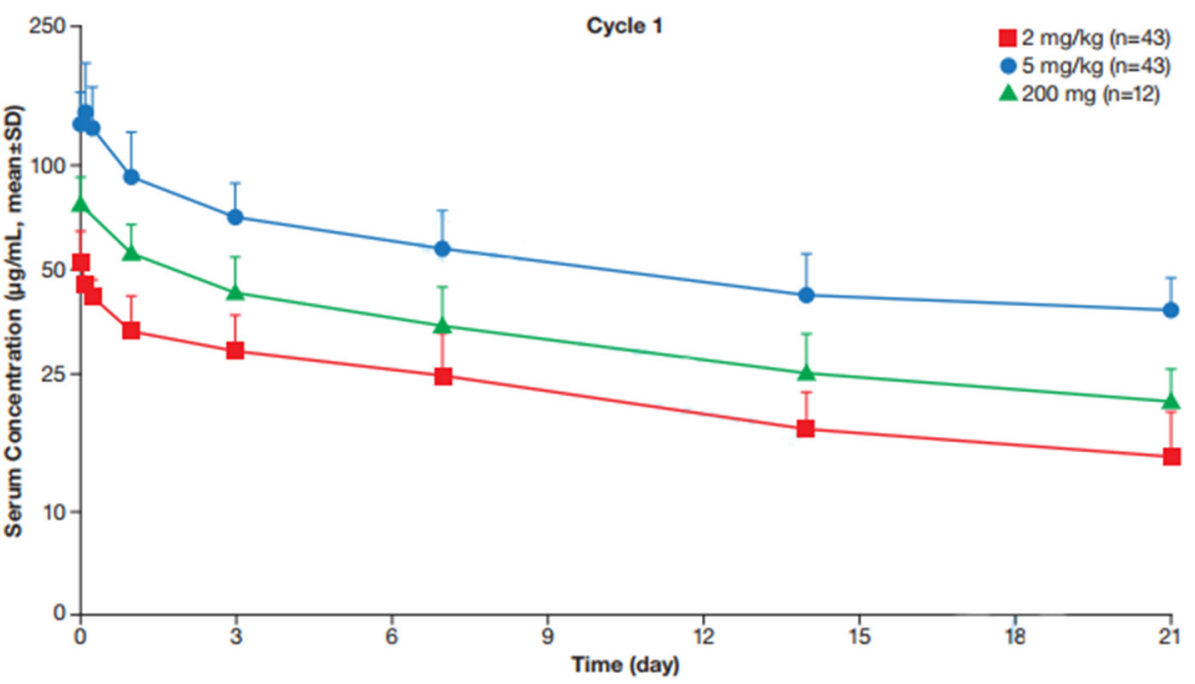

Figure 2 Concentration of tislelizumab following a single $200 \mathrm{mg}$ dose vs single 2 and $5 \mathrm{mg}$ dose.

categorized according to severity (NCI-CTCAE V.4.03), seriousness and relationship to the study treatment. Antitumor activity was assessed by CT imaging at screening, every 8 (every 2 weeks) or 9 (every 3 weeks) weeks for the first 12 months, and every 12 weeks thereafter. Tumor response was evaluated by RECIST V.1.1 criteria $^{13}$ for all tumor types. Formalin-fixed, paraffin-embedded tumor tissue from biopsies and resections collected before tislelizumab treatment were used for PD-L1 analysis by immunohistochemistry with the VENTANA PD-L1 (SP263) Assay (Ventana Medical Systems, Tucson, Arizona, USA). Definition of PD-L1 positivity was different for individual tumor types based on previously reported cut-offs. The rationale of cut-off selection for each indication was based on published PD-L1 algorithms. PD-L1 expression was scored as: 1) the percentage of tumor cells (TC) with any membrane staining; the percentage of tumor-associated immune cells (IC) with staining at any intensity or 3) the percentage of tumor area occupied by tumor-associated IC (TC/IC) with staining at any intensity (see online supplementary appendix, p3).

Standard PK parameters (eg, area under the concentration-time curve (AUC), maximal plasma concentration $\left(\mathrm{C}_{\max }\right)$ and time to maximal plasma concentration) were estimated. As an indication of target engagement, PD-1 receptor occupancy assay was centrally assessed on samples taken from select patients enrolled in arm 2 in phase IB. Immunogenic responses to tislelizumab were assessed for antidrug antibody (ADA) formation. Blood samples for PK, receptor occupancy and immunogenicity assessment were collected as described below.

In part 1 , cycles 1 and 4 , predose (within 1 hour before administration) and postdose $(0.5,1.5,6,24,72$ (or 96), 168 and 336 hours after the end of infusion) blood samples were collected on days 1, 2, 4 (or 5), 8 and 15 to assess the PK profile of tislelizumab. For additional cycles, blood samples were collected on day 1 of each cycle at the following time points: predose (within 1 hour before administration) and postdose (end infusion to $30 \mathrm{~min}$ ). In part 2, predose (within $60 \mathrm{~min}$ before the start of infusion) and postdose (within $30 \mathrm{~min}$ after the end of infusion) samples were collected at cycle 1 day 1 and day 15 (only for every 2 weeks), day 1 of cycle 2 , every subsequent cycle in the first 12 months and approximately every 6 months thereafter. Additional PK samples were collected at cycle 1 day 4 (or 5), day 8 , day 15 (only for every 3 weeks) and at the mandatory safety follow-up visit. In part 3, predose (trough, within 24 hours before the start of infusion) and postdose (within $30 \mathrm{~min}$ after the end of infusion) samples were collected on day 1 of cycle 1 , cycle 2, cycle 3 , cycle 5 and every two cycles in first 6 months, every four cycles in the next 6 months and approximately every 6 months thereafter. Additional PK samples were collected at day 2, day 4 (or 5), day 8 and day 15 of cycle 1 and cycle 5 ; a predose sample was also collected on cycle 5 day 22 (cycle 6 day 1). Further PK samples were collected at the mandatory safety follow-up visit.

For subjects in phase IA part 1, blood samples for immunogenic analysis were collected before starting the day 1 infusion every cycle in the first 6 months, every two cycles in the next 6 months, approximately every 6 months thereafter and at the mandatory safety follow-up visit. For subjects in phase IA part 2, blood samples were collected within 24 hours before starting the day 1 infusion every cycle in the first 6 months, every two cycles (every 2 weeks) or three cycles (every 3 weeks) in the next 6 months, approximately every 6 months thereafter and at the mandatory safety follow-up visit. For subjects in phase IA part 3, blood samples were collected before starting the day 1 infusion every two cycles in the first 6 months, every four cycles in the next 6 months, approximately every 6 months thereafter and at the mandatory safety follow-up visit. For subjects in phase IB, blood samples were collected before starting the day 1 infusion of cycle 1 and every two cycles in the first 6 months, every four cycles in the next 6 months, approximately every 6 months thereafter and at the mandatory safety follow-up 
Table 3 Treatment-emergent adverse events* (SAF; $n=451)$

\begin{tabular}{|c|c|c|c|c|}
\hline \multirow[b]{2}{*}{ Preferred term } & \multicolumn{4}{|c|}{ Total $(n=451)$} \\
\hline & Grade 1-2 & Grade 3 & Grade 4 & Grade 5 \\
\hline Fatigue & $120(26.6)$ & $8(1.8)$ & 0 & 0 \\
\hline Nausea & $106(23.5)$ & $6(1.3)$ & 0 & 0 \\
\hline Decreased appetite & $92(20.4)$ & 0 & 0 & 0 \\
\hline Constipation & 76 (16.9) & $3(0.7)$ & 0 & 0 \\
\hline Diarrhea & $75(16.4)$ & $8(1.8)$ & 0 & 0 \\
\hline Abdominal pain & $62(13.7)$ & $7(1.6)$ & 0 & 0 \\
\hline Cough & $62(13.7)$ & 0 & 0 & 0 \\
\hline Back pain & $61(13.5)$ & $6(1.3)$ & 0 & 0 \\
\hline Rash & $60(13.3)$ & $1(0.2)$ & 0 & 0 \\
\hline Vomiting & $58(12.9)$ & $8(1.8)$ & 0 & 0 \\
\hline Dyspnea & $43(9.5)$ & $2(0.4)$ & 0 & 0 \\
\hline Weight decreased & $37(8.2)$ & $3(0.7)$ & 0 & 0 \\
\hline Hypercalcemia & $19(4.2)$ & $3(0.7)$ & 0 & 0 \\
\hline Anemia & $19(4.2)$ & $22(4.9)$ & 0 & 0 \\
\hline Increased ALT & $17(3.8)$ & $8(1.8)$ & 0 & 0 \\
\hline Increased AST & $16(3.5)$ & $7(1.5)$ & 0 & 0 \\
\hline $\begin{array}{l}\text { Lower respiratory } \\
\text { tract infection }\end{array}$ & $12(2.7)$ & $3(0.7)$ & 0 & 0 \\
\hline Hypokalemia & $12(2.7)$ & $8(1.8)$ & $1(0.2)$ & 0 \\
\hline Dysphagia & $9(2.0)$ & $7(1.6)$ & 0 & 0 \\
\hline Pneumonia & $7(1.6)$ & $20(4.4)$ & 0 & $3(0.7)$ \\
\hline Pleural effusion & $7(1.6)$ & $5(1.1)$ & 0 & $1(0.2)$ \\
\hline Pneumonitis & $6(1.3)$ & $6(1.3)$ & 0 & $1(0.2)$ \\
\hline Hypertension & $6(1.3)$ & $6(1.3)$ & 0 & 0 \\
\hline Ascites & $6(1.3)$ & $7(1.6)$ & 0 & 0 \\
\hline Hyponatremia & $5(1.1)$ & $3(0.7)$ & 0 & 0 \\
\hline $\begin{array}{l}\text { Increased blood } \\
\text { bilirubin }\end{array}$ & $4(0.9)$ & $2(0.4)$ & $1(0.2)$ & 0 \\
\hline Colitis & $3(0.7)$ & $3(0.7)$ & 0 & 0 \\
\hline Hyperglycemia & $1(0.2)$ & $4(0.9)$ & $1(0.2)$ & 0 \\
\hline $\begin{array}{l}\text { Pulmonary } \\
\text { embolism }\end{array}$ & 0 & $5(1.1)$ & 0 & 0 \\
\hline $\begin{array}{l}\text { Upper } \\
\text { gastrointestinal } \\
\text { hemorrhage }\end{array}$ & 0 & $4(0.9)$ & 0 & 0 \\
\hline $\begin{array}{l}\text { Small intestinal } \\
\text { obstruction }\end{array}$ & 0 & $3(0.7)$ & 0 & 0 \\
\hline Sepsis & 0 & $1(0.2)$ & $2(0.4)$ & $1(0.2)$ \\
\hline
\end{tabular}

Data presented as $\mathrm{n}(\%)$.

${ }^{*}$ This table shows all grade 1 or 2 adverse events occurring in $\geq 10 \%$ of patients and grade $3-5$ events occurring in three or more patients. ALT, alanine aminotransferase; AST, aspartate aminotransferase; SAF, safety analysis set.

visit. The immunogenicity results were summarized using descriptive statistics by the number and percentage of patients who developed detectable ADA. The incidence of positive ADA and neutralizing ADA is reported for patients in the ADA analysis set.
For select patients in arm 2 in phase IB, blood samples for receptor occupancy assessment were taken on cycle 1 day 1 (predose) and day 2 (approximately 24 hours after the first dose) as well as predose on day 1 of cycles 2 and 3 .

\section{Statistical analyses}

SAS V.9.4 was used for statistical analyses. Descriptive statistics were used to summarize all study data. The KaplanMeier method estimated median time and 95\% CI for progression-free survival (PFS) and overall survival (OS). The safety/tolerability profile and antitumor activity of tislelizumab were assessed in the safety analysis set (SAF; patients who received at least one dose of tislelizumab); DLTs were determined from the DLT set (patients who experienced a DLT during cycle 1 and received at least $90 \%$ of the planned doses during the DLT observation period (cycle 1)) and the PK profile was established from the PK analysis set (patients who had received at least the first dose of tislelizumab and provided PK samples as per protocol following first dosing).

\section{RESULTS \\ Population disposition, demographics and disease baseline characteristics}

Between May 2015 and October 2017, 27 sites in Australia, Korea, New Zealand and Taiwan enrolled 451 patients (phase IA, $n=116$; phase IB, $n=335$ ) (see online supplementary figure S1). In phase IA part 1, 22 patients received one of four escalating doses of tislelizumab every 2 weeks $(0.5 \mathrm{mg} / \mathrm{kg}(\mathrm{n}=3), 2 \mathrm{mg} / \mathrm{kg} \quad(\mathrm{n}=6), 5 \mathrm{mg} / \mathrm{kg} \quad(\mathrm{n}=6)$ and $10 \mathrm{mg} / \mathrm{kg}(\mathrm{n}=7))$. In part 2,81 patients were treated with tislelizumab $(2 \mathrm{mg} / \mathrm{kg}$ every 2 weeks $(\mathrm{n}=20), 5 \mathrm{mg} /$ $\mathrm{kg}$ every 2 weeks $(\mathrm{n}=20), 2 \mathrm{mg} / \mathrm{kg}$ every 3 weeks $(\mathrm{n}=21)$ and $5 \mathrm{mg} / \mathrm{kg}$ every 3 weeks $(\mathrm{n}=20)$ ). In part 3, 13 patients were treated with fixed-dose tislelizumab $(200 \mathrm{mg}$ every 3 weeks). All 335 patients enrolled in phase IB received tislelizumab $5 \mathrm{mg} / \mathrm{kg}$ every 3 weeks.

Median age of all enrolled patients was 61.0 years (range: 18-81) with more male than female patients (table 1). This was a heavily pretreated population with $>95 \%$ of patients having received $\geq 1$ prior anticancer drug treatments, including platinum compounds $(66.1 \%)$, pyrimidine analogs $(50.6 \%)$, taxanes $(38.8 \%)$, anthracyclines $(25.9 \%)$ and protein kinase inhibitors $(17.5 \%)$.

The median treatment duration of tislelizumab was 2.8 months (range: $0.13-43.7)$; 65 patients $(14.4 \%)$ received tislelizumab for $\geq 12$ months. The median duration of study follow-up was 8.6 months (range: $0.1-40.8$ ) in all patients: 13.6 months (range: $0.7,40.8$ ) in phase IA and 7.6 months $(0.1-35.9)$ in phase IB. Of the 451 enrolled patients, $123(27.3 \%)$ experienced $\geq 1 \mathrm{AE}$ leading to dose modification (ie, treatment interruption or dose delay). Overall, infusion-related reactions $(\mathrm{n}=12 ; 2.7 \%)$ was the most common AE leading to dose modification (see online supplementary table S1). Treatment with tislelizumab was discontinued in 24 patients $(5.3 \%)$ due to 
Table 4 Clinical response as assessed by investigator by phase and overall (SAF)

\begin{tabular}{|c|c|c|c|}
\hline & $\begin{array}{l}\text { Phase IA } \\
(n=116)\end{array}$ & $\begin{array}{l}\text { Phase IB } \\
(n=335)\end{array}$ & $\begin{array}{l}\text { Overall } \\
(n=451)\end{array}$ \\
\hline \multicolumn{4}{|l|}{ ORR (CR, PR) } \\
\hline \multicolumn{4}{|c|}{ Best overall confirmed response, $\mathrm{n}(\%)$} \\
\hline $\mathrm{CR}$ & $4(3.4)$ & $2(0.6)$ & $6(1.3)$ \\
\hline PD & $48(41.4)$ & $152(45.4)$ & $200(44.3)$ \\
\hline NE & $1(0.9)$ & $6(1.8)$ & $7(1.6)$ \\
\hline Missing & $4(3.4)$ & 39 (11.6) & $43(9.5)$ \\
\hline \multicolumn{4}{|l|}{ DCR (CR, PR, SD) } \\
\hline \multicolumn{4}{|c|}{ CBR (CR, PR, durable SD*) } \\
\hline $\mathrm{n}(\%)$ & $35(30.2)$ & $82(24.5)$ & $117(25.9)$ \\
\hline Exact $95 \% \mathrm{Cl}$ & 22.00 to 39.39 & 19.97 to 29.45 & 21.96 to 30.25 \\
\hline \multicolumn{4}{|c|}{ Time to response (months) } \\
\hline $\mathrm{N}$ & 21 & 39 & 60 \\
\hline Median (range) & $2.2(1.8$ to 15.9$)$ & $2.2(1.3$ to 10.2$)$ & $2.2(1.3$ to 15.9$)$ \\
\hline
\end{tabular}

${ }^{*}$ Durable SD is defined as SD with duration $\geq 24$ weeks.

CBR, clinical benefit rate; CR, complete response; DCR, disease control rate; NE, not estimable; ORR, objective response rate; PD, progressive disease; PR, partial response; SAF, safety analysis set; SD, stable disease.

tislelizumab-related AEs, most commonly due to pneumonitis $(n=8 ; 1.8 \%)$. One DLT was observed during dose escalation (grade 3 colitis in the $5 \mathrm{mg} / \mathrm{kg}$ every 2 weeks cohort); the maximum administered dose was $10 \mathrm{mg} / \mathrm{kg}$ every 2 weeks.

\section{Pharmacokinetic profile of tislelizumab}

The single-dose and multiple-dose PK profiles of tislelizumab were characterized from 109 patients from phase IA (table 2). Mean steady-state PK profiles for the every 2 weeks and every 3 weeks dosing regimens are presented in online supplementary figure $\mathrm{S} 2$. Drug exposure $\left(\mathrm{C}_{\max }\right.$ and $\left.\mathrm{AUC}_{0-14}\right)$ increased in a dose-proportional manner from 0.5 to $10 \mathrm{mg} / \mathrm{kg}$ tislelizumab; no correlation was reported between clearance and baseline body weight, supporting fixed dosing of tislelizumab. At the $5 \mathrm{mg} / \mathrm{kg}$ dose, PD- 1 receptor occupancy was $>90 \%$.

Data from the $200 \mathrm{mg}$ every 3 weeks cohort demonstrated that tislelizumab concentrations after the first dose fell within 2 and $5 \mathrm{mg} / \mathrm{kg}$ dose range (figure 2). Tislelizumab demonstrated a 1.69-fold accumulation ratio, which was consistent with the estimated terminal half-life following tislelizumab $200 \mathrm{mg}$ every 3 weeks $\left(\mathrm{t}_{1 / 2}=16.8 \pm 5.5\right.$ days). Steady-state trough concentrations from patients with various tumor types in phase IB generally fell within a similar range of concentrations (see online supplementary figure S3), suggesting the tislelizumab PK profile is independent of tumor type.

\section{Tislelizumab safety/tolerability profile}

Of the 451 enrolled patients, 436 (96.7\%) experienced $\geq 1 \mathrm{AE}$. Safety/tolerability profiles were generally consistent across study phases as well as dose levels and schedules (see online supplementary tables S2-3). Commonly reported grade 1-2 AEs included fatigue, nausea and decreased appetite (table 3). Fatigue was the only AE considered related to treatment in $\geq 10 \%$ of the total population $(13.1 \%)$, and was the most commonly reported tislelizumab-related AE (TRAE) across doses and schedules (see online supplementary tables S4 and S5). Potential irAEs occurred in $35.0 \%$ of patients; irAEs occurring in $\geq 5 \%$ of patients were rash (all terms; $14.6 \%$ ), diarrhea $(6.9 \%)$ and hypothyroidism $(6.0 \%)$. The emergence of ADAs occurred in $18.7 \%$ of patients across both phases, with one patient $(0.3 \%)$ testing positive for neutralizing antibodies.

AEs of grade 3-4 severity, regardless of attribution, were reported in 198 patients $(43.9 \%)$; these AEs were consistently reported across both phases. Grade 3-4 AEs occurring in $\geq 2 \%$ of patients included anemia $(\mathrm{n}=22$; $4.9 \%)$, pneumonia $(\mathrm{n}=21 ; 4.7 \%)$ and hypokalemia $(\mathrm{n}=9$; $2.0 \%)$. Thirty-nine patients $(8.6 \%)$ experienced a grade 3-4 TRAE; increased alanine aminotransferase $(n=6$; $1.3 \%)$, pneumonitis $(\mathrm{n}=5 ; 1.1 \%)$, increased aspartate aminotransferase $(n=4 ; 0.9 \%)$, colitis $(n=3 ; 0.7 \%)$, diarrhea $(n=3 ; 0.7 \%)$ and fatigue $(n=3 ; 0.7 \%)$ were TRAEs of 


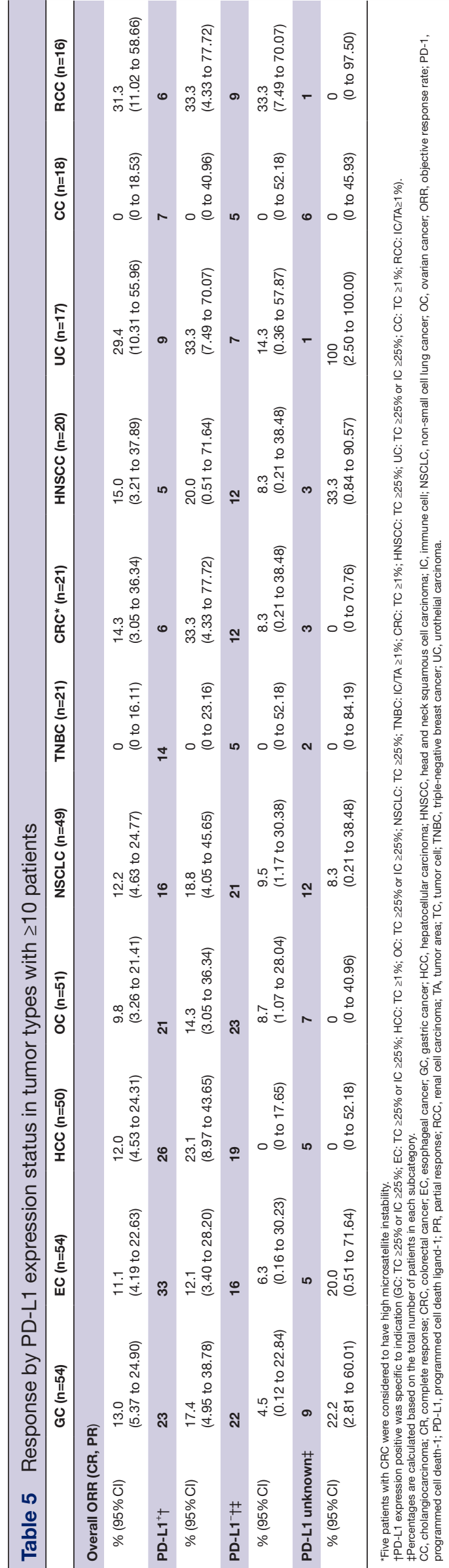

grade $3-4$ occurring in $>2$ patients. Serious AEs considered related to tislelizumab were reported in $35(7.8 \%)$ patients. Pneumonitis $(\mathrm{n}=8 ; 1.8 \%)$ and colitis $(\mathrm{n}=5$; $1.1 \%$ ) were the only serious AEs related to tislelizumab reported in $\geq 5$ patients. Overall, 14 patients $(3.1 \%)$ experienced a fatal AE. Two patients experienced fatal TRAEs (pneumonitis, $\mathrm{n}=1$; fulminant hepatitis, $\mathrm{n}=1$ ); both AEs were considered potentially immune-related and both cases were complicated by underlying disease. The pneumonitis event occurred in a patient with NSCLC with compromised pulmonary capacity at baseline; the hepatitis event occurred in a patient with hepatocellular carcinoma (HCC) with rapidly progressing disease.

\section{Antitumor activity of tislelizumab}

Confirmed responses were observed across phases (table 4) and dose/dosing schedules (see online supplementary table S6). Overall, the confirmed ORR was $13.3 \%$ (95\% CI: 10.31 to 16.79 ) (table 4). While responses were primarily driven by patients who achieved partial response $(\mathrm{PR} ; \mathrm{n}=54 ; 12.0 \%)$, six patients achieved complete response $(\mathrm{CR} ; 1.3 \%)$ in esophageal cancer, clear cell carcinoma of the endometrium, Merkel cell carcinoma, cutaneous squamous cell carcinoma, renal cell carcinoma and urothelial carcinoma $(\mathrm{n}=1 \mathrm{each})$. The overall disease control $(\mathrm{CR}+\mathrm{PR}+$ stable disease $(\mathrm{SD}))$ and clinical benefit $(\mathrm{CR}+\mathrm{PR}+\mathrm{SD} \geq 24$ weeks $)$ rates were $44.6 \%$ and $25.9 \%$, respectively. The median duration of response (DoR) was 16.0 months (95\% CI: 11.1 to 25.6). Median DoR was 14.6 months (95\% CI: 8.3 to not estimable (NE)) in phase IA and 16 months ( $95 \%$ CI: 9.2 to NE) in phase IB.

When responses per tumor type were evaluated by PD-L1 status using previously published cut-offs, there was a trend of higher ORRs in PD-L1-positive (PD-L1 ${ }^{+}$) patients with HCC, as well as gastric, esophageal, ovarian, non-small cell lung, colorectal, head and neck squamous cell and urothelial cancers (table 5). Notably, all patients in the HCC cohort who responded to tislelizumab were $\mathrm{PD}^{-} \mathrm{L1}^{+}(\mathrm{ORR}=23.1 \%)$. In patients with renal carcinoma, ORR was identical between PD-L1 ${ }^{+}$and $\mathrm{PD}-\mathrm{L}^{-}$groups $(33.3 \%$ vs $33.3 \%)$. No patients from the triple-negative breast cancer or cholangiocarcinoma cohorts responded to treatment, and thus the impact of PD-L1 status could not be assessed.

\section{Estimated survival associated with tislelizumab treatment}

As of the data cut-off of May 20, 2019, 58.3\% of patients with an objective response had progressed or died. Median OS across the study was 10.3 months (95\% CI: 8.5 to 11.6 ); median OS was 13.6 months (95\% CI: 9.9 to 17.5 ) and 9.3 months (95\% CI: 7.7 to 11.1 ) in phases IA and IB, respectively (see online supplementary figure S4A). Overall survival rates at 6 and 12 months were $67.3 \%$ (95\% CI: 62.7 to 71.6 ) and $43.1 \%$ (95\% CI: 38.3 to 47.8 ), respectively. Across the study, median PFS was 2.1 months (95\% CI: 2.1 to 2.7), with a median PFS of 3.5 months (95\% CI: 2.1 to 3.8) in phase IA and 2.1 months (95\% CI: 2.1 to 2.2) in phase IB. The proportion of patients with 
PFS at 6 and 12 months was $29.5 \%$ (95\% CI: 25.2 to 33.8 ) and $15.6 \%$ (95\% CI: 12.3 to 19.2 ), respectively (see online supplementary figure $\mathrm{S} 4 \mathrm{~B}$ ).

\section{DISCUSSION}

This phase IA/IB study was conducted to characterize the safety/tolerability, PK and preliminary antitumor activity of tislelizumab monotherapy. Across the 451 patients enrolled from Australia, Korea, New Zealand and Taiwan, tislelizumab was generally well tolerated with a safety/ tolerability profile consistent with other anti-PD-1 antibodies. $^{10}{ }^{14-19}$ The majority of tislelizumab-related AEs observed in this study were grade 1-2 in severity; $9 \%$ of patients had grade $\geq 3$ AEs considered possibly related to tislelizumab. irAEs were reported in $35 \%$ of patients; rash was the only reported irAE with an incidence of $>10 \%$. Severe and fatal treatment-related AEs were uncommon. Clinical responses were observed across multiple solid tumors. These responses were durable, with a median duration of 16 months.

A key study objective was to determine the appropriate dosing for future clinical studies. Ultimately, a fixed $200 \mathrm{mg}$ dose of tislelizumab every 3 weeks was selected for further evaluation based on comparable safety, PK and efficacy profiles between the $200 \mathrm{mg}$ fixed-dose and the 2 and $5 \mathrm{mg} / \mathrm{kg}$ weight-based doses. One DLT was observed (grade 3 colitis) in the $5 \mathrm{mg} / \mathrm{kg}$ every 2 weeks cohort. No clear dose-related safety/tolerability concerns were observed between patients receiving 2 and $5 \mathrm{mg} /$ $\mathrm{kg}$ every 2 weeks and every 3 weeks, and no unexpected tislelizumab-related AEs occurred in the $200 \mathrm{mg}$ fixeddose cohort compared with body weight-based cohorts, and the practicalities provided by fixed dosing. Additionally, tislelizumab clearance was found to be independent of body weight, ethnicity and sex; observed exposure of a $200 \mathrm{mg}$ dose fell within the bounds of serum exposure observed after 2 and $5 \mathrm{mg} / \mathrm{kg}$ doses. Finally, ORRs in patients treated with tislelizumab 2 and $5 \mathrm{mg} / \mathrm{kg}$ every 2 weeks ranged between $10 \%$ and $15 \%$, compared with a range of $15 \%-38 \%$ for patients treated at 2 and $5 \mathrm{mg} / \mathrm{kg}$ every 3 weeks. Of the evaluable patients treated at $200 \mathrm{mg}$ every 3 weeks $(n=13)$, three $(23 \%)$ had achieved partial response, consistent with results seen with the weightbased cohorts.

As these data are from a non-randomized, open-label study, the data have some inherent limitations. Response and survival of tislelizumab were assessed in a heterogeneous group of patients with a variety of advanced solid tumors with different levels and patterns of PD-L1 expression. Patients with $\mathrm{PD}-\mathrm{L1}^{-}$tumors achieved objective responses, suggesting that PD-L1 expression, as a single biomarker for response, may not be sufficient to identify those who are more likely to respond to checkpoint inhibition. It is important to note that key differences in PD-L1 antibody clones and various immunohistochemical platforms have raised questions about comparability. In terms of assessment of PD-L1 expression, specific tumor-type cutoffs vary depending on the antibody clone used. As different anti-PD-L1 antibody clones can have different levels of sensitivity and specificity, and can recognize different epitopes, using the same cut-off for different clones may cause results to be skewed, leading to an inappropriate treatment selection. Finally, the clinical impact of tislelizumab's lack of binding to Fc $\gamma$ RI compared with other anti-PD1 antibodies was difficult to determine in this phase IA/IB trial. Additional studies are needed to enhance our understanding of the tumor microenvironment and the effects of anti-PD- 1 antibodies and further evaluation of tislelizumab in specific tumor types will provide a more accurate assessment of its antitumor activity.

In this study, single-agent tislelizumab demonstrated a favorable safety/tolerability profile and induced durable clinical responses in $\geq 10 \%$ of patients, which is promising in this heavily pretreated population, including a balanced population of both Caucasian and Asian patients. Furthermore, these responses were observed in patients with either PD-L1 ${ }^{+}$or PD-L1 ${ }^{-}$tumors; additional studies are needed to further characterize the predictive value of PD-L1, and optimize a diagnostic/treatment algorithm for different tumor types. Based on the strength of these data, tislelizumab is currently being evaluated as monotherapy and in combination therapy for the treatment of solid tumors and hematological malignancies, including in registrational studies in esophageal, gastric, HCC, lung and urothelial cancers.

\section{Author affiliations}

${ }^{1}$ Peter MacCallum Cancer Centre, University of Melbourne, Melbourne, Victoria, Australia

${ }^{2}$ Auckland City Hospital, Auckland, New Zealand

${ }^{3}$ Seoul National University Bundang Hospital, Seongnam-si, South Korea

${ }^{4}$ National Taiwan University Hospital, Taipei, Taiwan

${ }^{5}$ National Cheng Kung University Hospital, Tainan, Taiwan

${ }^{6}$ Taipei Veterans General Hospital, Taipei, Taiwan

${ }^{7}$ Seoul National University Hospital, Seoul, South Korea

${ }^{8}$ Waikato Hospital, University of Auckland Waikato Clinical Campus, Hamilton, New Zealand

${ }^{9}$ Chang Gung Memorial Hospital, Chang Gung University, Linkou, Taiwan

${ }^{10}$ Asan Medical Center, Seoul, South Korea

${ }^{11}$ Monash Health, Monash University, Clayton, Victoria, Australia

${ }^{12}$ Chang Gung Memorial Hospital, Chia-yi, Taiwan

${ }^{13}$ Division of Pulmonary and Critical Care Medicine, Department of Internal Medicine, Kaohsiung Chang Gung Memorial Hospital, Kaohsiung, Taiwan

${ }^{14}$ Chris O'Brien Lifehouse, Sydney, New South Wales, Australia

${ }^{15}$ Sydney Medical School, University of Sydney, Sydney, New South Wales, Australia ${ }^{16}$ Department of Medical Oncology, Prince of Wales Hospital and Prince of Wales Clinical School, University of New South Wales, Sydney, New South Wales, Australia

${ }^{17}$ Tasman Oncology Research Ltd, Southport, Queensland, Australia

${ }^{18}$ BeiGene USA, Inc, San Mateo, California, USA

${ }^{19}$ BeiGene (Beijing) Co., Ltd, Beijing, China

${ }^{20}$ Linear Clinical Research, Nedlands, Western Australia, Australia

Acknowledgements The authors would like to thank the investigative center study staff, the study patients and their families. The authors would like to thank Regina Switzer, PhD and Elizabeth Hermans, PhD (OPEN Health Medical Communications, Chicago, Illinois, USA) for providing writing and editorial assistance.

Contributors Concept and design: JD, BM, MM. Provision of study materials or patients: all authors. Collection and assembly of data: all authors. Data analysis and 
interpretation: JD, YZ, LL, VP. Manuscript writing and critical evaluation: all authors. Final approval of manuscript: all authors. Accountable for all aspects of the work: all authors.

Funding The study protocol was developed by BeiGene, Ltd. in collaboration with the study investigators. BeiGene, Ltd. was also involved in data collection, analysis and interpretation of results. Statistical analyses were performed by statisticians at BeiGene, Ltd.

Competing interests JD has served in a consulting or advisory role for Amgen, BeiGene, Bionomics, Eisai, Eli Lilly and Novartis, and their institution has received research funding from Bionomics, GlaxoSmithKline, Novartis and Roche. SD has received personal fees from Roche; C-CL has served in a consulting or advisory role for Novartis and has received travel, accommodations and expenses from BeiGene; BK has received grants and personal fees from AstraZeneca and MSD Oncology, grants from Ono Pharmaceutical and personal fees from Genexin; MJ's institution has received research funding from BeiGene, Merck Sharp \& Dohme, Pfizer and Bristol-Myers Squibb; BM has served in a consultancy or advisory role for Novartis; LH has received grants from Astellas; MF has received honoraria from AstraZeneca, Merck Sharp \& Dohme, Lilly, Takeda and Novartis, serves in a consulting or advisory role for AstraZeneca, Merck Sharp \& Dohme and has received research funding from BeiGene and AstraZeneca. AH is employed by, has a leadership role in and has stock or other ownership in, Tasman Healthcare/Tasman Oncology Research. SS has received grants from Merck, Britsol-Meyers Squibb, Amgen, Endocyte, AstraZeneca and Roche; PB has received personal fees from Roche; LL, C-YW, JW, VP and YZ are employees of BeiGene; MM serves in a consulting or advisory role for AstraZeneca, Boehringer Ingelheim, Bristol-Myers Squibb, Merck Sharp \& Dohme, Novartis and Roche, and has received travel, accommodations and expenses from AstraZeneca, Bristol-Myers Squibb, Merck Sharp \& Dohme and Roche.

Patient consent for publication Not required.

Ethics approval This study was conducted according to the ethical principles of the Declaration of Helsinki, Good Clinical Practice guidelines, the principles of informed consent and the requirements of the public registration of clinical trials. Written informed consent was obtained from each patient prior to screening. The protocol was approved by the institutional ethics committee and was monitored by a safety monitoring committee.

Provenance and peer review Not commissioned; externally peer reviewed.

Data availability statement Data are available on reasonable request. On request, and subject to certain criteria, conditions and exceptions, BeiGene will provide access to individual de-identified participant data from BeiGene-sponsored global interventional clinical studies conducted for medicines (1) for indications that have been approved or (2) in programs that have been terminated. BeiGene will also consider requests for the protocol, data dictionary and statistical analysis plan. Data requests may be submitted to medicalinformation@beigene.com.

Open access This is an open access article distributed in accordance with the Creative Commons Attribution Non Commercial (CC BY-NC 4.0) license, which permits others to distribute, remix, adapt, build upon this work non-commercially, and license their derivative works on different terms, provided the original work is properly cited, appropriate credit is given, any changes made indicated, and the use is non-commercial. See http://creativecommons.org/licenses/by-nc/4.0/.

\section{REFERENCES}

1 Dahan R, Sega E, Engelhardt J, et al. FcyRs modulate the anti-tumor activity of antibodies targeting the PD-1/PD-L1 axis. Cancer Cell 2015;28:285-95.

2 Mahoney KM, Freeman GJ, McDermott DF. The next immunecheckpoint inhibitors: PD-1/PD-L1 blockade in melanoma. Clin Ther 2015;37:764-82.

3 Patel SP, Kurzrock R. PD-L1 expression as a predictive biomarker in cancer immunotherapy. Mol Cancer Ther 2015;14:847-56.

4 Herbst RS, Soria J-C, Kowanetz M, et al. Predictive correlates of response to the anti-PD-L1 antibody MPDL3280A in cancer patients. Nature 2014;515:563-7.

5 McDermott DF, Atkins MB. PD-1 as a potential target in cancer therapy. Cancer Med 2013;2:662-73.

6 Konishi J, Yamazaki K, Azuma M, et al. B7-H1 expression on non-small cell lung cancer cells and its relationship with tumorinfiltrating lymphocytes and their PD-1 expression. Clin Cancer Res 2004;10:5094-100

7 Shi F, Shi M, Zeng Z, et al. PD-1 and PD-L1 upregulation promotes CD8(+) T-cell apoptosis and postoperative recurrence in hepatocellular carcinoma patients. Int J Cancer 2011;128:887-96.

8 Wu C, Zhu Y, Jiang J, et al. Immunohistochemical localization of programmed death-1 ligand-1 (PD-L1) in gastric carcinoma and its clinical significance. Acta Histochem 2006;108:19-24.

9 Brahmer JR, Drake CG, Wollner I, et al. Phase I study of single-agent anti-programmed death-1 (MDX-1106) in refractory solid tumors: safety, clinical activity, pharmacodynamics, and immunologic correlates. J Clin Oncol 2010;28:3167-75.

10 Topalian SL, Hodi FS, Brahmer JR, et al. Safety, activity, and immune correlates of anti-PD-1 antibody in cancer. N Engl J Med 2012;366:2443-54

11 Hamid O, Robert C, Daud A, et al. Safety and tumor responses with lambrolizumab (anti-PD-1) in melanoma. N Engl J Med 2013;369:134-44.

12 Zhang T, Song X, Xu L, et al. The binding of an anti-PD-1 antibody to $\mathrm{Fc \gamma RI}$ has a profound impact on its biological functions. Cancer Immunol Immunother 2018;67:1079-90.

13 Eisenhauer EA, Therasse P, Bogaerts J, et al. New response evaluation criteria in solid tumours: revised RECIST guideline (version 1.1). Eur J Cancer 2009;45:228-47.

14 Fuchs CS, Doi T, Jang RW, et al. Safety and efficacy of pembrolizumab monotherapy in patients with previously treated advanced gastric and gastroesophageal junction cancer: phase 2 clinical KEYNOTE-059 trial. JAMA Oncol 2018;4:e180013.

15 Nanda R, Chow LQM, Dees EC, et al. Pembrolizumab in patients with advanced triple-negative breast cancer: phase Ib KEYNOTE-012 study. J Clin Oncol 2016;34:2460-7.

16 Bellmunt $\mathrm{J}$, de Wit R, Vaughn DJ, et al. Pembrolizumab as secondline therapy for advanced urothelial carcinoma. N Engl J Med 2017;376:1015-26.

17 Rosenberg JE, Hoffman-Censits J, Powles T, et al. Atezolizumab in patients with locally advanced and metastatic urothelial carcinoma who have progressed following treatment with platinum-based chemotherapy: a single-arm, multicentre, phase 2 trial. Lancet 2016;387:1909-20.

18 Rizvi NA, Mazières J, Planchard D, et al. Activity and safety of nivolumab, an anti-PD-1 immune checkpoint inhibitor, for patients with advanced, refractory squamous non-small-cell lung cancer (CheckMate 063): a phase 2, single-arm trial. Lancet Oncol 2015;16:257-65.

19 Powles T, O'Donnell PH, Massard C, et al. Efficacy and safety of durvalumab in locally advanced or metastatic urothelial carcinoma: updated results from a phase 1/2 open-label study. JAMA Oncol 2017;3:e172411. 